\title{
Speed and Torque Control of 3 Phase Induction Motors using Periferal Interface Controller
}

\author{
Jithin J*, K R Devika, Jasim Ali M, Krishnenedhu Murali \\ Electrical and Electronics Engineering, TKM College of Engineering Kollam, India
}

*Corresponding Author: Jithin J, Electrical and Electronics Engineering, TKM College of Engineering Kollam, India

\begin{abstract}
Controlling the speed of induction motors have tremendous use on various fields of engineering and technology. With the advancement in semiconductor fabrication technology, energy inefficient mechanical motor drives and control systems are replaced by variable frequency drives. VFDs can also improve the motor's steady state and dynamic characters. In this paper we are discussing the speed control of the motor keeping the voltage to frequency ratio constant. Periferal Interface Controller microcontroller, Insulated Gate Bipolar Transistors are used for achieving this and the result were simulated using MATLAB\&Simulink software
\end{abstract}

Keywords: 3phase induction motor, IGBTs, PIC microcontrollers, v/f ratio

\section{INTRODUCTION}

Efficient control of a three phase induction motor is a very tedious task. But with the advent of power electronics, it became less cumbersome. Power semiconductors with faster switching rate has made this possible. Induction motors when connected to 3 phase supply runs at rated speed. Due to the complexity in motor characteristics of a 3 phase induction motor, it is difficult to control the speed unlike a dc motor. But in so many fields, variable speed operation of the motor is necessary. Also less weight, low cost and lack of commutator makes the induction motors more advantageous over $\mathrm{dc}$ motors. In this paper we are using a 6 switch 3 phase $(6 \mathrm{~s} 3 \mathrm{ph})$ inverter for the control of speed and torque of the motor. We are using a Periferal Interface Controller (PIC) microcontroller for controlling the switching operation.

It is the time for electric vehicle to switch from brushless dc motors to induction motors. The speed control of the induction motors to be used in electric vehicles is used here. The dc supply from battery of the vehicle is converted to ac by the inverter and fed into the motor. For the practical easiness we have used a 3 phase supply from the main boards and converted back to dc using rectifier circuits. Additional circuits are also discussed for the smooth running of the motor.

\section{MOTOR DRIVES}

\subsection{Induction Motors}

In an induction motor, electric current in the rotor, needed to produce the required torque is obtained by electromagnetic induction from the magnetic field of stator winding which is connected to a three phase energy source.The operation of induction motors is based on the rotating magnetic field produced by the stator coils. It induces corresponding rotor current. This rotor current produces magnetic field that opposes the stator magnetic field according to Lenz's Law. Thus the rotor will start to rotate to reduce the change in rotor winding current. So induction motors do not require a commutator which eliminates the contact drops and have higher efficiency. Also reduced cost, high reliability, ease of operation, durability has replaced dc motor. They found many applications in chemical, fertilizer, automobile, cement industries.

\subsection{Speed Control by V/F Method}

The base speed of the motor is directly proportional to supply frequency and number of poles. By design the number of poles is constant. Thus, the speed is best varied by varying the supply 
frequency. Torque is directly proportional to voltage and frequency. For maintaining constant torque in an induction motor the ratio of voltage and frequency is kept constant, their values continuously changing throughout the speed range. Thus using V F control good range of speed adjustment is achieved in a simple and cost effective way.

\section{CírCuits AND COMPONENTS}

\subsection{Rectifiers}

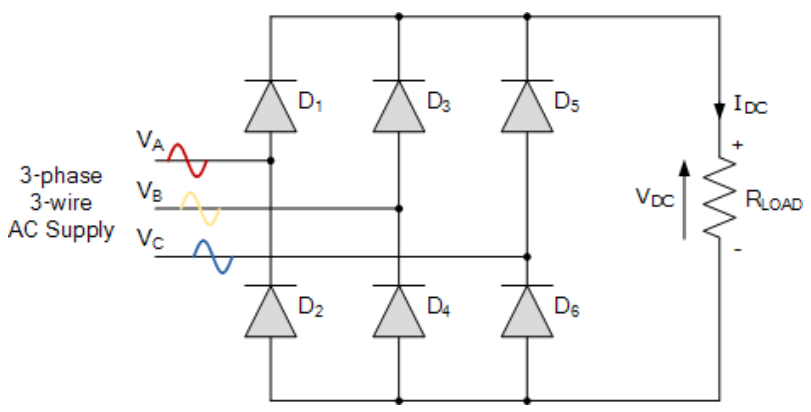

Fig1. Three phase rectifier circuit

Rectifier in three phase inverter converts the main ac single phase supply to a dc supply. By using capacitors, ripples in the output waveform is filtered and smoothened.

Rectifier section is made up of a group of silicon rectifiers. These can be a group of 6,18 or 24 . As 12 or 18 sets of diodes take up more space and cost more, we use 6 pulse converters. The reduced harmonic distortion in 12 or 18 pulse rectifiers can be achieved here by including an LC filter in the line side of the drives.

Silicon controller rectifier increase switching gradually, thus increasing the voltage applied to charge the DC bus. They are mounted on heat sink to control the generated heat during rectification. Convectional flow of air

provided by the fan on top removes the heat from sink effectively. This ensures increased life of rectifiers.

Figure 1 shows the rectification of three phase ac voltage. Diode ensures the unidirectional flow of current. During the positive cycle of the alternating voltage L1, the diode D1 conducts and D2 remain off whereas D2 conducts and D1 remain off in the negative cycle. Thus the direction of current flow will always happen from bottom to top. Similarly, the other phases also get rectified. Here the top line gets more positive and bottom gets negative.

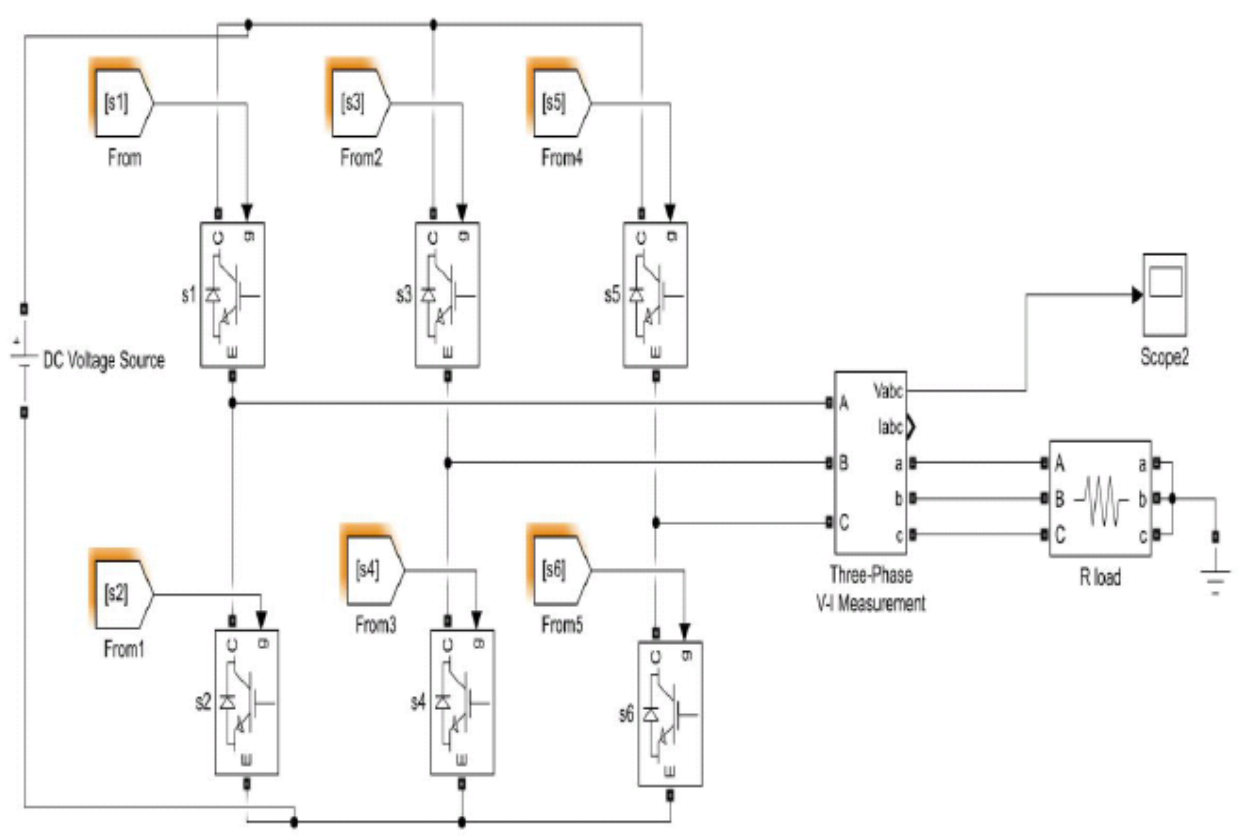

Fig2. Simulink model for dc to 3 phase ac inverter 


\subsection{Dc Link}

DC power from the rectifier section is fed to DC link. It consists of capacitors and inductors which smoothen ripples. To allow easier service, 3 bank arrangement of 12 capacitors in each is used. This helps in reducing harmonic noise. Their ability to store dc power helps to compensate temporary loss of power. DC link voltage is 1.35 times the input voltage.

\subsection{Inverter}

Fig.2. shows the inverter which converts dc power to 3 phase ac power by using 6 IGBTs as shown in the figure. The sin pwm as shown in fig.3. produced by the micro controller will feed to 6 IGBTs which will decide the switching of these IGBTs. The upper half cycle is controlled by IGBTs S1, S3 and S5 whereas lower half cycle is controlled by S2, S4 and S6

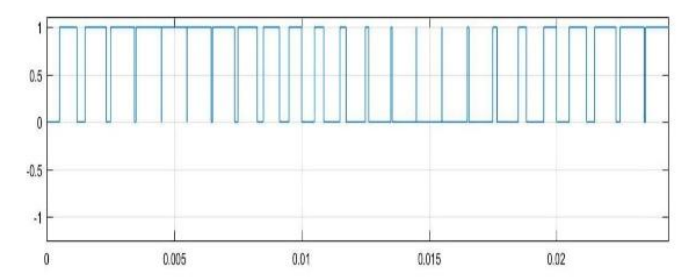

Fig3. Simulation of the sinpwm produced by the microcontroller using Matlab Simulink

The highlighted S1 in the figure represents these sinpwm signals to corresponding IGBT S1. The dc constant voltage source shown in the diagram is to be replaced by the three phase rectifier section as discussed earlier. The load represents the motor and three phase V-I measurement device measure the voltage being supplied to motor. Each pair of IGBTs represent one phase. In each phase, there is a phase difference of $120^{\circ}$. So this phase difference is made by suitable offsetting.

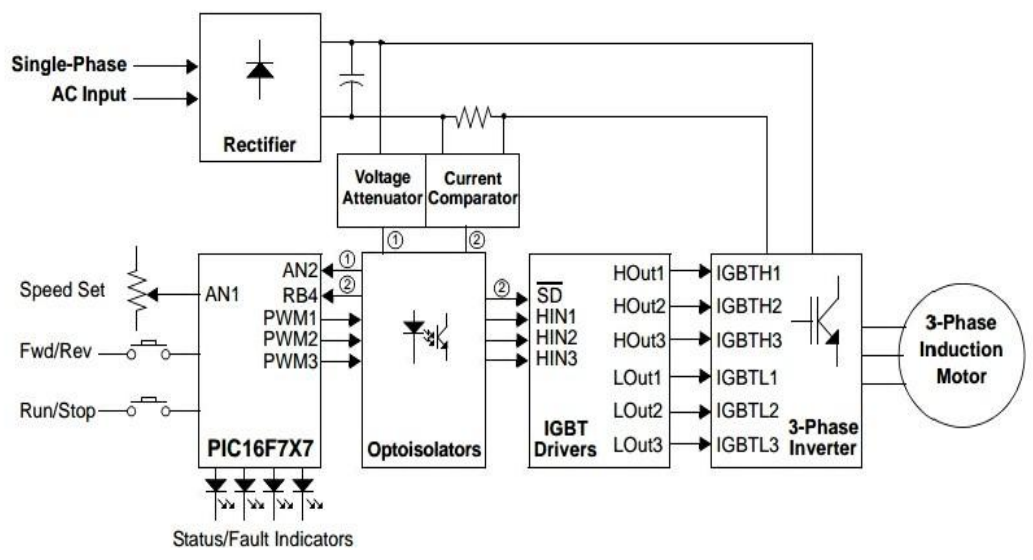

Fig4. Speed controller block diagram

The microcontroller used here is PIC16F727. It can produce 3 pwm at a time. The dutycycle and period of the each pwm can be controlled separately. For controlling 6 IGBTs using 3 pwm, the 3 pwm are fed into the IGBT driver as shown in Fig.4.IGBT driver produces two pwm for each of its input. So we get 6 pwm out of which 3 are sent to upper IGBTs and other 3 is inverted and given to lower IGBTs. There is a time lag between the signals given to the IGBTs for smooth production of 3 phase ac power in both positive and negative half cycles. These are also controlled by the IGBT driver.

\subsection{Brake Circuit}

In conveyors, and centrifuges, direction need to be changed quickly or needed to be stopped. Dynamic breaking is used in this situation. When motors having large inertia, is stopped or direction changed, it reduces power. For removing this power, additional transistor is used.

\subsection{Sensors}

The current going to each terminal of three phase motor is monitored through sensors. When short circuit is discovered, sensors detect and alarm. Constant monitoring helps in disconnection of motor from the drive when ground circuit is discovered. 


\subsection{LC Filters}

Output may not be sinusoidal due to switching time taken by the switches. Distortion of input voltage and current increases, as harmonics increases. Pulsating torque is produced which reduces the performance of three phase induction motor. To overcome this a passive filter is connected after inverter section of VFD. Thereby the performance of the motor is increased. Stator current, motor speed and electromagnetic torque waveform with less harmonic distortion in steady state conduction is obtained. This results in less pulsation electromagnetic torque

\section{Simulation Results}

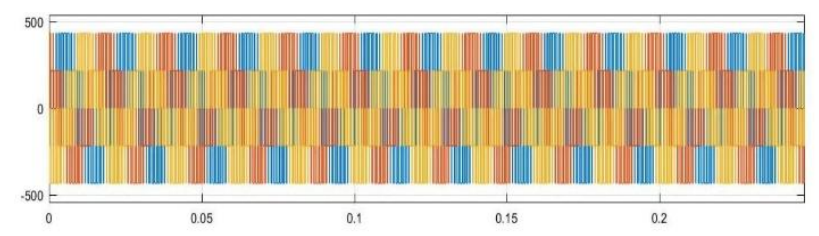

Fig.5. a.

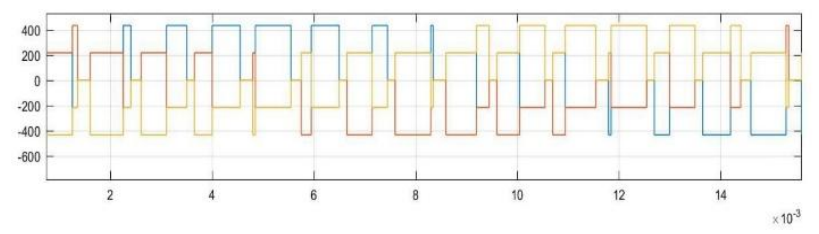

Fig.5. b.

Figures 5.a. and 5.b. shows the output voltages from the speed controller that is fed into the motor. Three phases are represented using the colours red, blue and yellow. Fig.5.b is the enlarged view of Fig.5. a. and the sinusoidal variation in voltages is clearly depicted.

\section{Conclusion}

The proposed model effectively deals with the speed and torque control of three phase induction motors. Use of IGBT enables faster switching at a high voltage which has a tremendous role in the efficiency of the inverter and thus the smooth functioning of the motor. This design improved motor's dynamic and steady state characteristics with reduced average energy consumption. Harmonic distortion is minimized in the circuit drastically. The performance of the model is analyzed through simulation using Matlab Simulink and found to be satisfactory. This method can be implemented in an Electrical vehicle or an Electric hybrid vehicle as a motor drive and can achieve the speed control.

\section{REFERENCES}

[1] D. Kumar, "Performance Analysis of Three-Phase Induction Motor with AC Direct and VFD," IOP Conference Series: Materials Science and Engineering, vol. 331, p. 012025, 2018.

[2] K. Srinivasan and S. Dash, "Digital simulation of low cost fuzzy logic based four-switch three-phase (PWM) inverter fed induction motor drive," IET-UK International Conference on Information and Communication Technology in Electrical Sciences (ICTES 2007), 2007.

[3] R. V. Tiede, "Speed-torque control of induction motors," Electrical Engineering, vol. 69, no. 5, pp. 409409, 1950.

[4] J. Schofield, "Variable speed drives using induction motors and direct torque control," IEE Colloquium on Vector Control Revisited, 1998.

Citation: Jithin J, et.al. (2019). "Speed and Torque Control of 3 Phase Induction Motors using Periferal Interface Controller". International Journal of Research Studies in Electrical and Electronics Engineering (IJRSEEE), 5(3), pp 1-4. DOI: http://dx.doi. org/10.20431/2454-9436.0503001

Copyright: (c) 2019 Authors. This is an open-access article distributed under the terms of the Creative Commons Attribution License, which permits unrestricted use, distribution, and reproduction in any medium, provided the original author and source are credited. 